\title{
artigo
}

\section{A enfermagem no contexto da hemoterapia: a segurança ao paciente}

\author{
Nursing in the context of hemotherapy: safety for patients \\ Enfermería en el contexto de la hemoterapia: seguridad para los pacientes
}

\begin{abstract}
RESUMO
Objetivo: Descrever o conhecimento dos enfermeiros no que se refere às práticas hemoterápicas e a segurança do paciente no processo. Métodos: Estudo descritivo, de abordagem direta e de caráter qualitativo, com 20 enfermeiros de um hospital privado de médio porte do município de Divinópolis, Minas Gerais. Foi realizado no período de agosto de 2018 a setembro de 2018 com aplicação de questionário. Resultados: A partir da análise das respostas dos participantes da pesquisa foi possível a criação de quatro categorias temáticas; Práticas transfusionais: treinamentos, atualizações e impressos que auxiliem na hemotransfusão; Checagem das informações necessárias para realização das transfusões; Monitorização do paciente no processo transfusional; Reações adversas e notificação. Conclusão: 0 estudo evidenciou profissionais cientes da importância do procedimento, porém observou-se falhas em alguns dos temas, vendo-se a necessidade de atualização, treinamentos e maiores pesquisas na área, a fim de buscar a segurança do paciente e qualidade do atendimento.
\end{abstract}

DESCRITORES: Segurança do paciente; Serviço de hemoterapia; Transfusão de sangue.

\section{ABSTRACT}

Objective: To describe the nurses' knowledge regarding hemotherapeutic practices and patient safety in the process. Methods: Descriptive study, with a direct and qualitative approach, with 20 nurses from a medium-sized private hospital in the municipality of Divinópolis, Minas Gerais. It was carried out from August 2018 to September 2018 with the application of a questionnaire. Results: From the analysis of the responses of the research participants, it was possible to create four thematic categories; Transfusion practices: training, updates and forms that assist in blood transfusion; Checking the information needed to perform the transfusions; Monitoring the patient in the transfusion process; Adverse reactions and notification. Conclusion: The study showed professionals aware of the importance of the procedure, but there were flaws in some of the themes, seeing the need for updating, training and further research in the area, in order to seek patient safety and quality of care.

DESCRIPTORS: Hemotherapy service; Patient safety; Blood transfusion.

\section{RESUMEN}

Objetivo: Describir el conocimiento de las enfermeras sobre las prácticas hemoterapéuticas y la seguridad del paciente en el proceso. Métodos: Estudio descriptivo, con enfoque directo y cualitativo, con 20 enfermeras de un hospital privado de tamaño mediano en el municipio de Divinópolis, Minas Gerais. Se realizó entre agosto de 2018 y septiembre de 2018 con la aplicación de un cuestionario. Resultados: Del análisis de las respuestas de los participantes en la investigación, fue posible crear cuatro categorías temáticas; Prácticas de transfusión: capacitación, actualizaciones y formularios que ayudan en la transfusión de sangre; Verificar la información necesaria para realizar las transfusiones; Monitoreo del paciente en el proceso de transfusión; Reacciones adversas y notificación. Conclusión: El estudio mostró a los profesionales conscientes de la importancia del procedimiento, pero hubo fallas en algunos de los temas, al ver la necesidad de actualización, capacitación e investigación adicional en el área, a fin de buscar la seguridad del paciente y la calidad de la atención.

DESCRIPTORES: Seguridad del paciente; Servicio de hemoterapia; Transfusión de sangre.

RECEBIDO EM: 23/07/2020 APROVADO EM: 28/07/2020

\section{Daniela Ribeiro Lopes}

Enfermeira. Graduada em Enfermagem pela Universidade do Estado de Minas Gerais (UEMG), Unidade Divinópolis.

ORCID: 0000-0002-1968-9114 


\section{Isabela Ribeiro Gontijo}

Enfermeira. Graduada em Enfermagem pela Universidade do Estado de Minas Gerais (UEMG), Unidade Divinópolis. ORCID: 0000-0002-2281-481X

\section{Marla Ariana Silva}

Graduanda em Enfermagem pela Universidade do Estado de Minas Gerais (UEMG), Unidade Divinópolis. ORCID: 0000-0003-0136-7122

\section{Fernanda Marcelino de Rezende e Silva}

Enfermeira. Doutoranda em Ciências da Saúde pela Universidade Federal de São João Del Rei (UFSJ), Campus Centro Oeste Dona Lindu (CCO). Docente e Coordenadora do Curso de Graduação em Enfermagem da Universidade do Estado de Minas Gerais (UEMG), Unidade Divinópolis.

ORCID: 0000-0003-2236-7009

\section{Silmara Nunes Andrade}

Enfermeira. Doutora em Ciências da Saúde pela Universidade Federal de São João Del Rei (UFSJ), Campus Centro Oeste Dona Lindu (CCO). Docente do Curso de Graduação em Enfermagem da Universidade do Estado de Minas Gerais (UEMG), Unidade Divinópolis.

ORCID: 0000-0002-1975-0827

\section{Regina Consolação dos Santos}

Enfermeira. Mestre em Ciência pela Universidade Federal de São João Del Rei (UFSJ), Campus Centro Oeste Dona Lindu (CCO). Docente do Curso de Graduação em Enfermagem da Universidade do Estado de Minas Gerais (UEMG), Unidade Divinópolis. Docente e coordenadora do curso de Enfermagem da Universidade de Itaúna (UIT), Minas Gerais.

ORCID: 0000-0002-7393-3210

\section{INTRODUÇÃO}

A hemoterapia é um processo dinâmico e complexo que tem como alicerce a Lei $n^{\circ} 10.205$, de 21 de março de 2001 que institui sobre as etapas do ciclo do sangue, sendo elas: coleta, processamento, estocagem, distribuição e aplicação do sangue ${ }^{(1)}$. Após a realização da coleta do sangue total, este é encaminhado para o setor de fracionamento, para um processo de centrifugação e fracionamento, no qual será obtido os hemocomponentes do sangue (concentrado de hemácias, concentrado de plaquetas e o plasma fresco congelado). Todos os sangues passam por testes sorológicos para a detecção de patógenos a fim de impedir a transmissão de doenças ${ }^{(2)}$.

Nas instituições hospitalares o componente mais utilizado nas transfusões é o concentrado de hemácias $(\mathrm{CH})$, um hemocomponente obtido através do fracionamento de uma bolsa de sangue total e que tem como indicação principal a melhora da oxigenação dos tecidos do organismo (3).

Para o adequando funcionamento e garantia de um processo seguro são uti- lizados normas e legislações editadas pelas autoridades sanitárias brasileiras, a fim de garantir ao máximo a segurança do paciente e a qualidade do serviço(4). No Brasil as práticas hemoterápicas são regulamentadas atualmente pela Portaria $\mathrm{n}^{\circ} 158$, de 4 de fevereiro de 2016, que redefine o regulamento técnico de procedimentos hemoterápicos ${ }^{(5)}$.

Mesmo se tratando de um processo que segue normas e indicações, a hemoterapia pode ocasionar possíveis incidentes, mesmo após a realização de todas as exigências e constante monitorização, devendo ser feita em condições seguras, por profissionais capacitados, com recursos necessários, a fim de evitar eventuais intercorrências ${ }^{(6)}$.

Dessa forma, torna-se imprescindível que o enfermeiro tenha conhecimentos amplos e habilidades técnicas, pautados na ética, no embasamento científico e no compromisso com a saúde dos indivíduos, favorecendo a diminuição de ocorrências de erros e garantindo a segurança transfusional ${ }^{(7)}$.

A equipe de enfermagem executa suas atribuições de acordo com o disposto em legislação específica pautada pela Lei $n^{\circ}$ 7.498, de 25 de junho de 1986, e o Decre- to $\mathrm{n}^{\circ} 94.406$, de 08 de junho de 1987 , que regulamenta o exercício da Enfermagem no país. Ainda é ressaltado que compete ao enfermeiro cuidados de enfermagem de maior complexidade técnica e que exijam conhecimentos científicos adequados e capacidade de tomar decisões imediatas ${ }^{(8)}$.

A Resolução do Conselho Federal de Enfermagem (COFEN) no 306/2006, estabelece como competências do enfermeiro o: "planejamento, execução, coordenação, supervisão e avaliação dos procedimentos hemoterápicos e de enfermagem nas unidades, visando assegurar a qualidade do sangue e hemocomponentes/hemoderivados coletados e transfundidos" ${ }^{\prime(9)}$.

$\mathrm{O}$ enfermeiro juntamente com a equipe possui papel de destaque por realizar a comunicação e interlocução direta com o paciente e por atuar diretamente no cuidado, e por isto deve buscar meios e ações que garantam e promovam a segurança ao paciente. A Classificação Internacional de Segurança do Paciente define 'segurança do paciente' como o ato de evitar, prevenir ou melhorar os resultados adversos ou as lesões originadas no processo de atendimento médico hospitalar ${ }^{(10)}$. 


\section{artigo}

Levando em consideração que se trata de um procedimento que, quando realizado de forma incorreta, pode acarretar consequências graves e até mesmo oferecer risco de morte, a realização deste estudo justificou-se pela relevância do tema e pela importante participação da enfermagem na área, além de ter possibilitado produção científica sobre a temática e capacitação para atuação. Diante disso, o estudo apresenta a seguinte pergunta norteadora: $\mathrm{O}$ enfermeiro que atua diretamente com as práticas hemoterápicas possui habilidades técnicas e cientificas para garantir a segurança ao paciente?

O objetivo deste estudo é descrever o conhecimento dos enfermeiros no que se refere às práticas hemoterápicas e a segurança do paciente no processo.

\section{MÉTODOS}

Trata-se de um estudo descritivo, de abordagem direta e de caráter qualitativo. A pesquisa buscou descrever e analisar o conhecimento dos profissionais de maneira fiel, sendo feito uma decomposição dos dados e na interpretação buscou-se sentidos nas falas e ações para chegar a uma compreensão ou explicação além do descrito ${ }^{(11)}$. Foi utilizado também o método de saturação teórica, no qual houve a suspensão da inclusão de novos participantes quando a coleta de dados passou a apresentar redundância ou repetição na avaliação do pesquisador ${ }^{(12)}$.

O estudo foi realizado em uma Instituição Hospitalar Particular de médio porte de referência do município de Divinópolis, Minas Gerais. A amostra total foi de 25 participantes, porém, só participaram 20 enfermeiros, dentre eles supervisores e assistenciais. Cinco enfermeiros de recusaram a participar. O quesito principal era profissionais atuantes na área e que possuíam contato direto com os pacientes em terapia transfusional. Como critérios de exclusão não foram entrevistados enfermeiros em treining (treinamento introdutório), licença maternidade/médica, enfermeiros que atuam em setores sem a prática transfusional e que se recusaram a participar da pesquisa.

A etapa de coleta de dados foi realizada entre agosto de 2018 a setembro de 2018 sendo operacionalizada por meio de aplicação de questionário para aquisição de informaçóes. Os participantes foram convidados a responder as perguntas, o qual foi aplicado de acordo com a disponibilidade dos participantes. No momento do contato foi feito o esclarecimento do objetivo do estudo e também quanto à forma de condução do mesmo. No momento da coleta alguns enfermeiros se recusaram a participar por estarem ocupados e não foi disponibilizado pela instituição a coleta de dados da equipe da noite.

Os dados obtidos através do questionário foram transcritos, analisados e com base em estudos foi feita a interpretação das respostas. O material foi analisado profundamente e suas respostas deram origem a categorias e temas que responderão à pergunta norteadora.

O presente estudo obedeceu a Resolução CNS/CONEP 466 de 12 de dezembro de 2012 que aprova as diretrizes e normas de pesquisas envolvendo seres humanos ${ }^{(13)}$. Os dados somente foram coletados após a aprovação da pesquisa pelo comitê de ética em pesquisa da Universidade do Estado de Minas Gerais (COEP/UEMG), aprovado com número de CAAE: 94514218.4.0000.5115.

No momento da aplicação do questionário foi disponibilizado para leitura e assinatura, o Termo de Consentimento Livre e Esclarecido (TCLE), igualmente assinado, em duas vias de mesmo teor. Para manter sigilo, foi utilizado códigos para identificação dos profissionais, exemplo: E1, tendo por objetivo defender os interesses dos participantes da pesquisa em sua integridade e dignidade.

\section{RESULTADOS}

O estudo contou com a participação de 20 enfermeiros e com a análise das respostas foi possível a organização dos dados em 5 categorias temáticas: Práticas transfusionais: treinamentos, atualizações e impressos que auxiliem na hemotransfusão; Conhecimento dos profissionais quanto aos cuidados com a bolsa de concentrado de hemácia $(\mathrm{CH})$; Checagem das informações necessá- rias para realização da transfusão; Monitorização do paciente no processo transfusional; Reações adversas e notificação.

\section{Práticas transfusionais: treinamen- tos, atualizações e impressos que au- xiliem na hemotransfusão}

A primeira categoria reuniu perguntas relacionadas ao preparo do profissional para a realização da prática transfusional, tendo como primeiro tema os treinamentos que são oferecidos pela instituição.

Sempre estamos em treinamentos,
voltado para prática quando é ne-
cessário e há dúvidas (E2).
Sim, os treinamentos são bastante
frequentes (E1).
Sim, mais ou menos 3 vezes no ano(E7).

O segundo tema foi direcionado ao conhecimento da equipe quanto a um impresso que instrua o profissional na hora da realização do procedimento, que nesta instituição é denominado Instrução de Trabalho (IT). Os profissionais demonstraram ciência da existência da IT e consciência da sua importância, uma vez que ela minimiza significativamente os riscos ao paciente e nos momentos de dúvidas evita que os profissionais realizem procedimentos com insegurança.

Sim; toda vez que haver dúvidas, buscar seu conhecimento para realizar o procedimento (E2).

Sim, a IT é importante pois contribui para sanar possiveis dúvidas que possam surgir durante a realização do procedimento (E6). Simparapadronizar, evitar erros epromover a segurança do paciente (E11).

\section{Conhecimento dos profissionais quan- to aos cuidados com a bolsa de $\mathrm{CH}$}

Nesta categoria buscou-se observar aspectos relacionados ao cuidado com a bolsa de $\mathrm{CH}$. No primeiro tema foi questionado o tempo máximo que esta pode ficar fora da geladeira antes do início da sua administração. As entrevistas revelaram que grande parte dos profissionais tem 
uma ideia errônea quanto ao tempo, o que aumenta as chances de procedimentos que possam causar danos à saúde.

10 a 5 minutos ambientada (E1). 3 horas (E3).

De 30 a 40 minutos (E6).

5 minutos (E12).

Por 4 horas (E14).

No segundo tema questionou aos profissionais o tempo máximo, mínimo e ideal de transfusão. Foi demonstrando, em sua totalidade, conhecimento, preocupação e atenção dos profissionais para esta etapa do procedimento.

Máximo 4 horas e minimo 2 horas (E2). Tempo minimo de 2 horas para evitar edema agudo de pulmão, sobrecarga cardíaca. Tempo máximo de 4 horas para evitar contaminação bacteriana (E7).

Sim, 24 gotas. Após 10 minutos passa para 48 gotas (E16).

\section{Checagem das informações necessá- rias para realização da transfusão}

$\mathrm{Na}$ terceira categoria, levantou-se sobre esta checagem e conferência das informações contidas na bolsa e no rotulo antes do início da transfusão. $O$ primeiro tema foi sobre a dupla checagem das informações, quem realiza e para quais informações deve-se atentar.

É realizado uma dupla checagem com enfermeiros ou técnicos de enfermagem, conferindo o nome e 0 tipo sanguineo (E10).

Com os dados do prontuário, de identificação do paciente como nome, tipo sanguineo, componentes que estão sendo transfundidos (E11).

$A$ supervisão vai até a agência transfusional, confere a bolsa, o cartão e caderno de registro. Depois no setor a supervisão confere novamente com o profissional e os dois assinam no cartão e na folha de registro (dupla checagem) (E14).

O segundo tema foi direcionado as informações que devem ser questionadas ao paciente (quando possível) na beira do leito e comparadas a bolsa no momento exato da instalação do sangue antes do início da transfusão.

Verificar placa de identificação do paciente, e conferir se realmente é ele (E2). Prescrição médica como: nome completo, idade, nome da mãe, registro número do prontuário, número da bolsa, hemocomponentes, $A B O e$ $R H$ vindos do doador, número de bolsas prescritas e responsável pela dispensação (E5).

Nome do paciente, se já realizou transfusão sanguinea antes (E11).

Nome do paciente, leito, número de registro, data de nascimento e data (E14)

Nos recortes é evidenciado que o nome sempre é questionado ao paciente e alguns dos funcionários mencionaram perguntar outras informações como o tipo sanguíneo, atitudes importantes que minimizam erros além de passar confiança e tranquilidade ao paciente.

\section{Monitorização do paciente no proces- so transfusional}

Nesta etapa buscou-se questionar os profissionais quanto a esta supervisão, avaliação e anotação dos sinais vitais do paciente durante o processo de transfusão. Sabemos que a verificação dos sinais vitais garante uma avaliação melhor do paciente, evita complicações e a sua anotação proporciona respaldo aos profissionais em diversas situações. O primeiro tema é sobre a verificação dos sinais vitais antes e após o início da transfusão e qual o tempo ideal para esta verificação.

Sim, no ato da transfusão. Alerta para febre (E2).

Sim, antes de retirar bolsa da geladei$r a$, antes da transfusão e 10 minutos após iniciar a transfusão" (E4).

Sim, aferir assim que for iniciar (ES). Aferir para iniciar, 10 minutos após início e ao término ou se necessário pois no CTI ficam monitorados (E6).
A monitorização durante o transcurso da transfusão também é citada na Portaria de Consolidação e foi uma questão que foi levantada aos profissionais. Neste tema perguntamos aos profissionais a que se deve atentar durante esta monitorização.

Sim. Deve-se atentar ao aumento de temperatura, frequência cardíaca, respiratória e variações acentuadas de pressão arterial (E3).

Os sinais vitais e qualquer reação apresentado durante e após a hemotransfusão (náuseas, vomito, eritema, dispneia e taquicardia) (E8).

Sim, deve-se verificar frequência cardíaca, frequência respiratória, nivel de consciência, se não vai apresentar alguma reação tipo urticaria, prurido, hipotensão ou hipertensão, nivel de saturação, dentre outros. Atentar sempre para o acesso venoso, calibre, hipertermia (E14).

Como já citado anteriormente a evolução de enfermagem garante uma avaliação segura do paciente e respaldo aos profissionais, por isto, as instituições, devem exigir de toda equipe prontuários atualizados com todos os procedimentos realizados inclusive a administração de hemocomponentes. Este tema apresenta recortes de respostas dos profissionais quanto esta evolução em fichas e prontuário.

Sim, em impresso próprio e na evolução de enfermagem, além da checagem na prescrição médica (E5).

Sim, evolução de enfermagem $e$ RQ488 que é o formulário de registro transfusionais (E8).

\section{Reações adversas e notificação}

As perguntas realizadas nesta categoria buscaram questionar os participantes quanto as reações transfusionais e suas condutas e o primeiro tema perguntou se os profissionais entrevistados se sentem preparado para a identificação precoce das reações. As respostas obtidas nos fizeram questionar, de modo negativo, o preparo dos profissionais e um certo descaso com o tema em questão. 
Você sabe identificar quando você acompanha sua transfusão (E2).

Sim, e para agir (ES).

Algumas sim (E13).

Já no segundo tema foi questionado se os profissionais tinham conhecimento da existência e importância da FEAT (ficha de notificação e investigação de eventos adversos transfusionais não infecciosos imediatos e tardios). Percebe-se que muitos dos profissionais tem conhecimento da FEAT e da sua importância.

Sim, ela contribui para identificar qualquer incidente imediato ou tardio. A fim de evitar danos à saúde do cliente (E10).

Sim, porque se houver alguma reação, nessa ficha consta todos os dados necessários que possa realizar rastreamento sobre o doador (E14). Sim. A importância para investigar o que causou reação no cliente, motivos e fatos (E15).

No terceiro tema da categoria perguntou-se sobre o preenchimento da FEAT nos casos de uma reação adversa. Esta é uma informação que deve ser do conhecimento de toda equipe, para que seja dado a correta continuidade do serviço neste momento.

O supervisor responsável (E1).

Enfermeiro do setor ou coordenador (E9). $O$ profissional responsável pelo procedimento, ou médico responsável (E13).

\section{DISCUSSÃO}

O tema segurança do paciente tem sido muito evidenciado e constitui um grande desafio para a saúde ${ }^{(14)}$. Os riscos fazem parte do serviço e está ligado a diversos fatores, e como destaque a carência de capacitação ${ }^{(16)}$. A Resolução do COFEN no ${ }^{\circ}$ 306/2006 destaca que o enfermeiro é responsável por proporcionar condições para o aprimoramento dos profissionais através de cursos, treinamentos e atualizações além de desenvolver pesquisas relacionadas à hemoterapia e impressos que padronizem o procedimento na instituição afim de um serviço mais seguro e de confiança ${ }^{(16)}$.

Segundo Junior e Rattner ${ }^{(17)}$, as práticas transfusionais atualmente são práticas seguras, porém pequenas falhas podem oferecer riscos à saúde dos pacientes, como o processamento, armazenado e administração do sangue em tempo e ambientes que possam comprometer a sua esterilidade. Visando a segurança do paciente é necessário atentar para que o início da transfusão não exceda 30 minutos após o recebimento da bolsa, pois a inadequada manipulação do produto e a excessiva exposição a temperatura ambiente pode aumentar o risco de danos ${ }^{(8)}$.

A realização da transfusão sanguínea conta com dois momentos distintos de checagem que busca minimizar ao máximo a chance de erros como, troca de bolsa, validade e incompatibilidade sanguínea ${ }^{(18-19)}$. A prevenção de acidentes durante o processo transfusional se inicia com a checagem das informações contidas no rotulo da bolsa e com a identificação correta do paciente. De acordo com Mattia ${ }^{(20)}$, a enfermagem não apenas realiza a administração, mas também deve conhecer suas indicações, realizar a checagem dos dados corretamente e estar preparada para orientar o paciente durante a transfusão.

Já Tavares ${ }^{(21)}$ em outro estudo ressalta a importância da equipe de enfermagem em atentar-se para a instalação correta do sangue, sem erros na identificação, realizar a dupla checagem, conhecer as indicações de transfusões, orientar os pacientes quanto ao procedimento e atender às reações adversas relacionadas a transfusão.

A Portaria de Consolidação № 5 do MS no Art. $19^{(8)}$ declara que o receptor, deverá ser identificado imediatamente antes da transfusão por meio do seu nome completo, prestada pelo próprio receptor ou por profissional da equipe e que deverá existir outros mecanismos para esta identificação como pulseias/braceletes em casos de pacientes impossibilitados. É ressaltada a suspensão da transfusão quando identificado erros de informações.
De acordo com Ribeiro ${ }^{(22)}$, a monitorização do paciente durante a transfusão pode ser considerada como a etapa mais importante, pois é nela que mudanças e alterações podem ser observadas e por isto é a que demanda maior compromisso e atenção. A equipe de enfermagem representa papel importante nesta etapa pois é a responsável pela administração dos hemocomponentes e supervisão durante o procedimento.

O Art. 190 da Portaria de Consolidação $\mathrm{N}^{\circ} 5$ do $\mathrm{MS}^{(8)}$ orienta que o paciente deve ter os seus sinais vitais (temperatura, pressão arterial e pulso) todos verificados e registrados imediatamente antes do início e após o término da transfusão e o paciente deve ser acompanhado pelo médico/profissional capacitado durante os primeiros 10 minutos. Estes registros devem conter data, hora de início e término da infusão, assim como sinais vitais antes durante $\mathrm{e}$ depois, identificação das bolsas dos hemocomponentes e do profissional responsável, dados da monitorização nos primeiros 10 minutos e durante o transcurso do ato transfusional ${ }^{(23)}$.

As reações transfusionais são agravos que podem ocorrer durante ou após a transfusão de sangue, tendo sinais e sintomas percebidos logo no início da transfusão ou até 24 horas após o procedimento ${ }^{(20)}$. Estas reações exigem ação imediata, tomada de decisão e estabelecimento de prioridades, a fim de que danos e desconforto sejam minimizados ${ }^{(24)}$.

\section{CONCLUSÃO}

Observou-se fragilidade, desencontros e desconhecimento em alguns dos temas abordados, evidenciando a necessidade de atualização e de treinamentos periódicos, a fim de enriquecer estes profissionais com o saber científico e técnico para a realização de um procedimento de qualidade e assim garantir a segurança do paciente. Por se tratar de um procedimento que pode oferecer risco a saúde, vê se a necessidade de mais estudos e pesquisas na área, a fim de levar mais conhecimentos aos profissionais tornando-os aptos a lidar com as diversas situações do procedimento. 
1. Cherem EO, Alves VH, Rodrigues DP, Souza FDL, Guerra JVV, Maciel VL. Saberes do enfermeiro para o cuidado no processo transfusional em recém-nascidos. Rev. Gaúcha Enferm. [internet]. 2017 [acesso em 30 jun. 2020]; 38(1). Disponível em: https://doi.org/10.1590/1983-1447.2017.01.63557.

2. Silva PAR, Assis DCM, Silva CR. Conhecimento de profissionais de enfermagem sobre atuação em hemotransfusão. Rev. Ciên. Saúde. 2017; 2(2): 15-24.

3. Anvisa. Consulta pública $n^{\circ} 37,20 / 05 / 2004$. Diretrizes para transfusão de hemácias.

4. Silva KFN, Soares S, Iwamoto HH. A prática transfusional e a formação dos profissionais de saúde. Rev. Bras. Hematol. Hemoter. 2009; 31(6): 421-426.

5. Brasil. Ministério da saúde. Portaria n 158 , de 4 de fevereiro de 2016. Redefine o regulamento técnico de procedimentos hemoterápicos. [acesso em 12 set. 2018]. Disponivel em: https://bvsms.saude.gov.br/bvs/saudelegis/ gm/2016/prt0158_04_02_2016.html

6. Ribeiro IP, Cabral LAF, Almeida AMLC, Silva TB. Perfil das hemotransfusões realizadas em um hospital de ensino de Teresina-PI. R. Inderd. 2013; 6(1): 88-95.

7. Faquetti MM, Rosa RL, Bellaguarda MLR, Lazzari DD, Tholl AD, Moraes CLK. Percepção dos receptores sanguíneos quanto ao processo transfusional. Rev. bras. Enferm. [internet]. 2014 [acesso em 30 jun. 2020]; 67(6). Disponível em: https://doi.org/10.1590/0034-7167.2014670611.

8. Brasil. Conselho Federal de Enfermagem. Resolução COFEN n511/2016. Norma técnica para atuação dos enfermeiros e técnicos de enfermagem em hemoterapia. [internet]. Rio de Janeiro: 2016. [acesso 30 jun. 2020]. Disponivel em: http://www.cofen.gov.br/resolucao-cofen-no-05112016_39095.html.

9. Brasil. Conselho Federal de Enfermagem. Resolução COFEN $n^{\circ}$ 306/2006. Normatiza a atuação do Enfermeiro em Hemoterapia. [internet]. Rio de Janeiro. 2006 abril. [acesso 30 jun 2020]. Disponivel em: http://www.cofen.gov.br/resoluo-cofen-3062006_4341.html.

10. Matiello RDC, Lima EFA, Coelho MCR, Oliveira ERA, Leite FMC, Primo CC. A cultura de segurança do paciente na perspectiva do enfermeiro. Rev. Cogitare Enferm. 2015; 20(3): 179-185.

11. Minayo MCS. Pesquisa social: teoria, método e criatividade. Petrópolis, RJ. 2010; 29.

12. Fontanella BJB, Júnior RM. Saturação teórica em pesquisas qualitativas: contribuições psicanalíticas. Rev. Psicologia em estudo. [internet]. 2012 [acesso em 30 jun. 2020]; 17(1): 63-71. Disponivel em: https://doi.org/10.1590/0034-71672016-0616.

13. Brasil. Ministério da Saúde. Conselho Nacional de Saúde. Resolução n 466, de 12 de dezembro de 2012. Diretrizes e normas regulamentadoras de pesquisas envolvendo seres humanos. [internet]. 2012. [acesso 30 jun. 2020]. Disponível em: https://bvsms.saude.gov.br/bvs/saudelegis/cns/2013/ res0466_12_12_2012.html

14. Vargas MAO, Luz AMH. Práticas seguras do/no cuidado de enfermagem no contexto hospitalar: é preciso pensar sobre isso e aquilo. Enferm. Foco. 2010; 1(1): 23-27.

15. Reis GAX, Hayakawa LY, Murassaki ACY, Matsuda LM, Gabriel CS, Oliveira MLF, et al. Implantação das estratégias de segurança do paciente: percepções de enfermeiros gestores. Texto contexto Enferm. [internet]. 2017 [acesso em 30 jun 2020]; 26(2): 1-9. Disponível em: https://doi. org/10.1590/0104-07072017000340016

16. Junior JBS, Rattner DA. Vigilância Sanitária no controle de riscos potenciais em serviços de hemoterapia no Brasil. Saúde debate. [internet]. 2016 [acesso em 30 jun. 2020]; 40(109). Disponivel em: https://doi.org/10.1590/01031104201610911

17. Brasil. Ministério da saúde. Portaria de Consolidação $n^{\circ}$ 05/2017. Consolidação das normas sobre as ações e os serviços de saúde do Sistema Único de Saúde, 2017. [acesso em 30 jun. 2020]. Disponivel em: https://portalarquivos2. saude.gov.br/images/pdf/2018/marco/29/PRC-5-Portariade-Consolida----0-n---5--de-28-de-setembro-de-2017. pdf.

18. Forster $\mathrm{F}$ et al. Percepção dos enfermeiros quanto à assistência de enfermagem no processo transfusional. Enferm em foco. 2018; 9(3): 71-75.

19. Reis VN et al. Monitorização transfusional: análise da prática assistencial em um hospital público de ensino. Einstein. 2016; 14(1): 41-46.

20. Mattia D, Andrade SR. Cuidados de enfermagem na transfusão de sangue: um instrumento para monitorização do paciente. Texto contexto-Enferm. [internet]. 2016 [acesso em 30 jun. 2020]; 25(2). Disponivel em: http://dx.doi. org/10.1590/0104-07072016002600015.

21. Tavares JL, Barichello E, Mattia AL, Barbosa MH. Fatores associados ao conhecimento da equipe de enfermagem de um hospital de ensino sobre hemotransfusão. Rev. Latino-Am. Enfermagem. 2015 [acesso em 30 jun. 2020]; 23(4). Disponível em: http://doi.org/10.1590/01041169.0024.2593.

22. Ribeiro IP, Cabral LAF, Almeida AMLC, Silva TB. Perfil das hemotransfusões realizadas em um hospital de ensino de Teresina-PI. R. Inderd. 2013; 6(1): 88-95.

23. Brasil. Ministério da Saúde. Agência Nacional de Vigilância Sanitária, Hemovigilância: Manual técnico de hemovigilância: investigação das reações. 2016; 25(2).

24. Grandi JL, et al. Hemovigilância: a experiência da notificação de reações transfusionais em Hospital Universitário. Rev. Esc. Enferm. USP. [internet]. 2018 [acesso em 30 jun. 2020]; 52(1). Disponível em: https://doi.org/10.1590/ s1980-220x2017010603331. 